\title{
Extreme multiexciton emission from deterministically assembled single-emitter subwavelength plasmonic patch antennas
}

\author{
Amit Raj Dhawan ${ }^{1,2}$, Cherif Belacel ${ }^{2,3}$, Juan Uriel Esparza-Villa ${ }^{2}$, Michel Nasilowski ${ }^{4}$, Zhiming Wang ${ }^{1}$, Catherine Schwob²,
} Jean-Paul Hugonin ${ }^{5}$, Laurent Coolen ${ }^{2}$, Benoît Dubertret ${ }^{4}$, Pascale Senellart ${ }^{3}$ and Agnès Maître $\mathbb{~}^{2}$

\begin{abstract}
Coupling nano-emitters to plasmonic antennas is a key milestone for the development of nanoscale quantum light sources. One challenge, however, is the precise nanoscale positioning of the emitter in the structure. Here, we present a laser etching protocol that deterministically positions a single colloidal CdSe/CdS core/shell quantum dot emitter inside a subwavelength plasmonic patch antenna with three-dimensional nanoscale control. By exploiting the properties of metal-insulator-metal structures at the nanoscale, the fabricated single-emitter antenna exhibits a very high-Purcell factor (>72) and a brightness enhancement of a factor of 70 . Due to the unprecedented quenching of Auger processes and the strong acceleration of the multiexciton emission, more than 4 photons per pulse can be emitted by a single quantum dot, thus increasing the device yield. Our technology can be applied to a wide range of photonic nanostructures and emitters, paving the way for scalable and reliable fabrication of ultracompact light sources.
\end{abstract}

\section{Introduction}

The interaction between an emitter and its local electromagnetic field can be engineered by increasing the local density of states for applications in quantum information $^{1}$ and single-photon generation ${ }^{2}$. This approach has been widely explored with a large variety of dielectric $^{3-5}$ or plasmonic environments ${ }^{6-10}$ and solid-state emitters such as self-assembled ${ }^{11}$ or colloidal ${ }^{12}$ quantum dots (QDs), single molecules ${ }^{13}$, and defects in diamond ${ }^{14}$. Achieving an intense light-matter interaction using extremely small mode volume plasmonic structures ${ }^{12,15}$ requires very precise, and hence challenging, spatial positioning of the emitter at the nanoscale. A large majority of the works so far have relied on randomly

\footnotetext{
Correspondence: Agnès Maître (agnes.maitre@insp.upmc.fr)

${ }^{1}$ Institute of Fundamental and Frontier Sciences, University of Electronic

Science and Technology of China, Chengdu 610054, People's Republic of China

${ }^{2}$ Sorbonne Université, CNRS, Institut des Nanosciences de Paris, UMR 7588,

75005 Paris, France

Full list of author information is available at the end of the article.
}

positioned emitters to demonstrate strong acceleration of the spontaneous emission of single emitters coupled to plasmonic antennas ${ }^{16-18}$. The challenge of deterministically positioning individual emitters inside nanophotonic structures with three-dimensional nanoscale control has to be overcome to realize efficient devices operating at room temperature and benefiting from highly optimized light-matter interaction regimes ${ }^{16,19}$.

Several approaches have been developed to precisely position single self-assembled quantum dots grown by molecular beam-epitaxy in cavities ${ }^{20-23}$. These approaches rely on optically measuring the QD position through emission mapping with subwavelength accuracy and defining the photonic structure around the QD using optical $^{20-22}$ or electronic ${ }^{23}$ lithography. However, these techniques cannot be extended to chemically synthetized emitters such as colloidal QDs or molecules that have strong potential for inexpensive and room-temperature applications. The main reason for this limitation is the exacerbated sensitivity of these emitters to technological

\section{(c) The Author(s) 2020}

(c) (i) Open Access This article is licensed under a Creative Commons Attribution 4.0 International License, which permits use, sharing, adaptation, distribution and reproduction cc) in any medium or format, as long as you give appropriate credit to the original author(s) and the source, provide a link to the Creative Commons license, and indicate if changes were made. The images or other third party material in this article are included in the article's Creative Commons license, unless indicated otherwise in a credit line to the material. If material is not included in the article's Creative Commons license and your intended use is not permitted by statutory regulation or exceeds the permitted use, you will need to obtain permission directly from the copyright holder. To view a copy of this license, visit http://creativecommons.org/licenses/by/4.0/. 
processing. Because of the high sensitivity of the emission process to surface states, preserving the properties of the colloidal QDs during technological protocols, involving, for example, e-beam or laser exposure, dry or wet etchings and solvents, is highly challenging.

Here, we report a non-destructive in situ far-field laser etching lithography technique that allows control-at the nanoscale-of the position of fragile emitters in plasmonic structures. Our technique employs multilayer structures and a dual-wavelength protocol to deterministically position a single $\mathrm{CdSe} / \mathrm{CdS}$ colloidal QD in a plasmonic patch antenna and fabricate subwavelength antennas of different shapes. As a result, we deterministically achieve a high Purcell factor for a single emitter. Strong electromagnetic interactions in the plasmonic antenna decrease the relative efficiency of the Auger recombination channels, which results in very high brightness and extreme multiexciton radiative recombination. The technique can be used to fabricate a variety of photonic structures embedding fragile emitters.

\section{Results}

\section{Plasmonic patch antenna for spontaneous emission} control

Optimizing the coupling between an emitter and a nanostructure makes it possible to control the emission directivity and the dynamics of the spontaneous emission. The latter is quantified by the Purcell factor $F_{\mathrm{P}}$, which scales as the inverse of the electromagnetic confinement volume provided by the photonic structure ${ }^{4,24,25}$. Plasmonic nanoantennas ${ }^{6}$ exhibit a very low mode volume and a wide spectral resonance and therefore are excellent structures for obtaining high Purcell factors $F_{\mathrm{P}}$ with broadband emitters.

Figure 1a depicts the system that is explored in the present work: a single emitter coupled to a plasmonic patch antenna. The antenna consists of a thin dielectric layer (typically $30-40 \mathrm{~nm}$ ) sandwiched between an optically thick bottom layer of gold and a thin gold patch on top; the patch thickness is on the order of $20 \mathrm{~nm}$, and its diameter is in the range of $0.2-2.5 \mu \mathrm{m}$. This system has been both theoretically and experimentally shown to be an excellent tool for accelerating and directing fluorescence emission ${ }^{26,27}$.

The optimal positioning of the emitter inside the antenna couples the radiation of the emitter to surface plasmon polaritons (SPPs) at both nano-spaced metaldielectric interfaces, and the SPPs in the patch are confined by the geometry of the patch. These SPPs at the two interfaces further couple and create strong confinement of the electromagnetic field around the emitter. The SPPs generated in the thin plasmonic metal patch (thinner than the skin depth) lead to the emission of photons $^{26}$ as depicted in Fig. 1a. The antenna operation depends on the dipole orientation of the emitter (stronger acceleration of the spontaneous emission for the vertical dipole orientation), the patch size (large patches are more directive, but small patches show stronger resonances), and the dielectric spacer ${ }^{26}$. In this work, we insert chemically synthetized ${ }^{28}$ relatively large quasi-spherical individual $\mathrm{CdSe} / \mathrm{CdS}$ semiconductor core/shell colloidal QDs ${ }^{29,30}$ in the antenna. These QDs have CdSe cores that are $\sim 3 \mathrm{~nm}$ in diameter and encapsulated by slowly grown CdS shells with a thickness of 6-8 nm (Fig. 1b), which make them almost nonblinking $^{31}$. Due to their high absorption cross-section, these QDs exhibit bright fluorescence at room temperature. Under ultra-violet (UV) excitation at room temperature, they emit at $633 \mathrm{~nm}$ with a spectral width of $30 \mathrm{~nm}$. We characterize these QDs in the lowexcitation limit, where Auger processes are efficient enough to lead to single-photon emission. We note that under strong pumping, these large single QDs emit multiple photons because their multiexciton emission rate becomes comparable to the non-radiative Auger rate (see Supplementary Information).
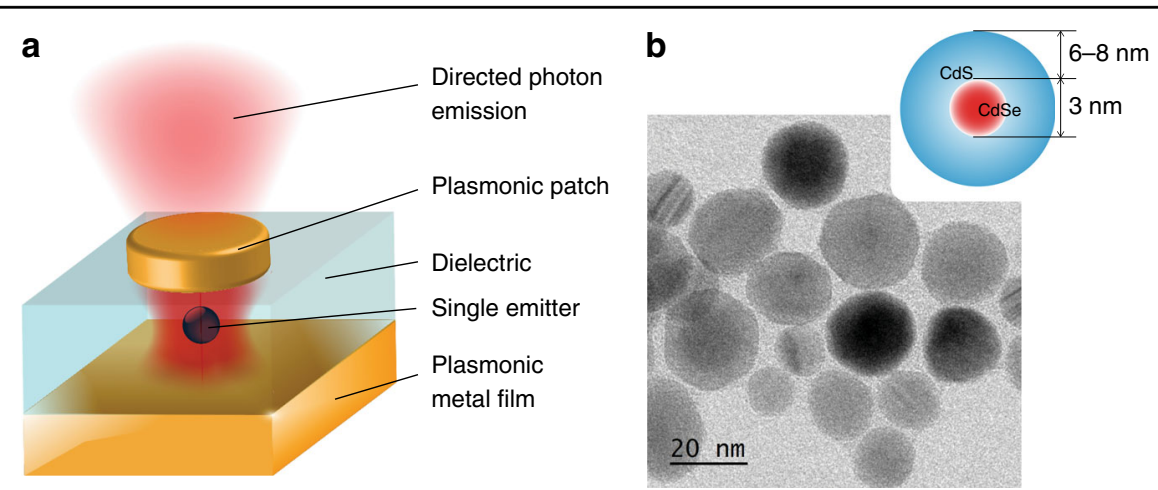

Fig. 1 A plasmonic patch antenna coupled to a single colloidal quantum dot. a Schematic of the structure under study consisting of a single colloidal core/shell QD coupled to a plasmonic patch antenna. b Schematic and transmission electron microscopy image of the investigated emitters 


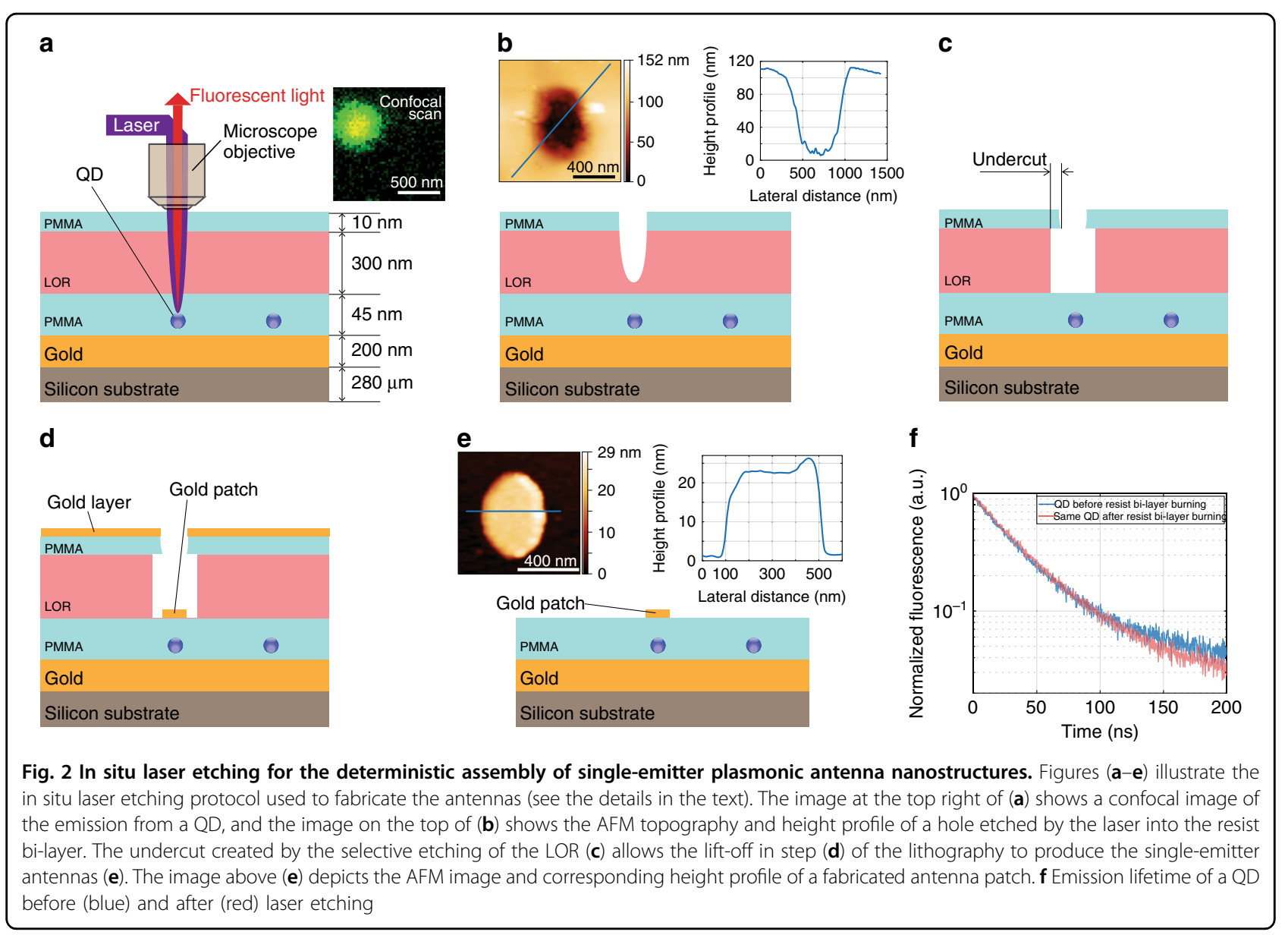

In situ subwavelength laser etching on fragile emitters

The proposed technological protocol is illustrated in Fig. 2 and consists of deterministic and non-destructive in situ laser etching, which allows the positioning of a single QD within an antenna with a $3 \mathrm{~nm}$ vertical precision and a $50 \mathrm{~nm}$ lateral precision (see Supplementary Information). We use a low-luminescence bi-layer polymer $^{32}$ to locate single emitters by mapping their luminescence.

The process starts by evaporating a thick layer of gold $(200 \mathrm{~nm})$ on a Si substrate using an intermediate adhesion layer of $\mathrm{Ti} / \mathrm{Cr}$. A 10 -nm thick layer of polymethyl methacrylate (PMMA) is then spin-coated above gold. Then, a layer of spatially well-isolated single QDs is spincoated using a dispersion of QDs in hexane of an appropriate dilution. To create an optimized spacing around the single emitters and to protect them from direct dielectric vapour deposition, a 35-nm thick smooth PMMA film is spin-coated, which embeds the emitters in a dielectric layer. This protects the emitters in the proceeding lithographic steps. A bi-layer consisting of lift-off resist (LOR) and PMMA is then spin-coated. The low luminosity of this bi-layer permits the detection of the emission from the single QDs embedded beneath and excited by a low-intensity spectrally filtered broadband supercontinuum laser emitting at 473-478 nm (Fig. 2a). As in the case of in situ lithography methods ${ }^{20,23}$, we map the QD fluorescence with nanoscale accuracy. The laser wavelength is then tuned to $550-605 \mathrm{~nm}$, which corresponds to a wavelength range where the laser light is absorbed substantially more by the lift-off resist than by the QD. The excitation power can thus be chosen to burn the resist bi-layer and locally create a hole directly above the selected QD without photobleaching the QD (Fig. 2b). We note in Fig. 2f, the emission lifetime of a QD does not change after the resist burns-this demonstrates that the laser etching process does not photodegrade the QD. After selective chemical etching of the LOR, an undercut is created in the PMMA (Fig. 2c). Finally, gold is deposited by evaporation (Fig. 2d), and a lift-off step is performed to obtain patch antennas embedding a single emitter (Fig. 2e). The undercut in the PMMA leads to a discontinuous deposition of gold (thus making the patch), and in step (e), the undercut provides a passage for the chemical etching agent to attack the LOR and liftoff the layers above the LOR while leaving the patch. The 
a

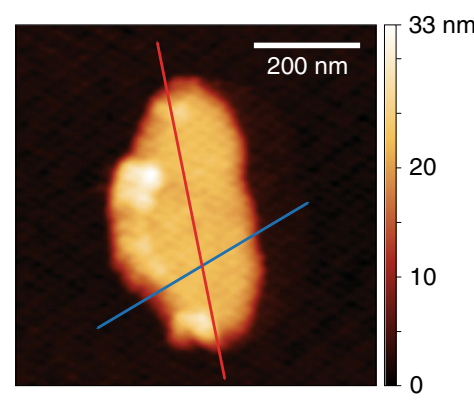

b

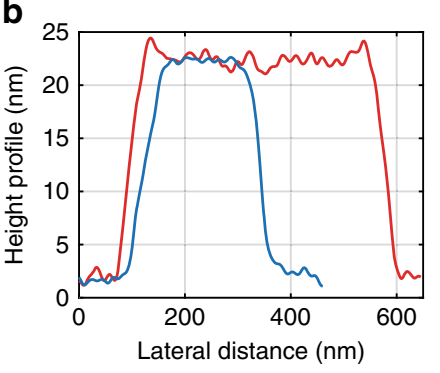

C

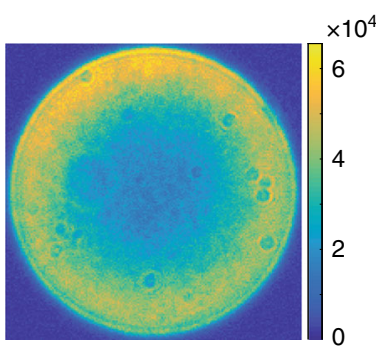

d

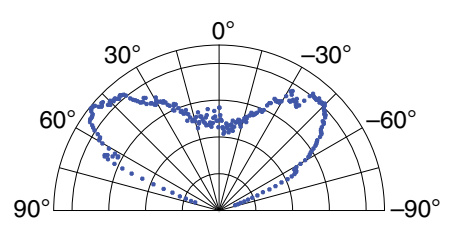

e

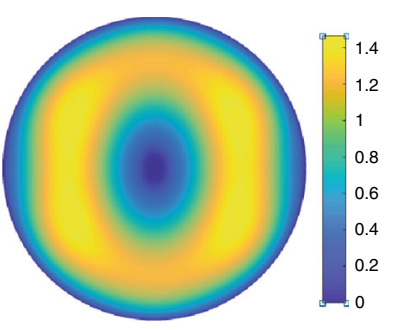

f

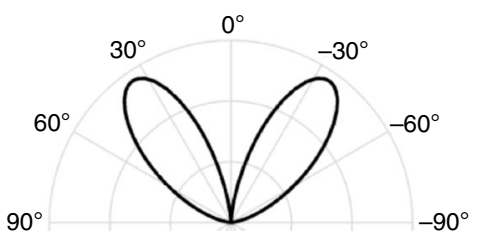

Fig. 3 Topography and emission pattern of the emitter-antenna structures. a AFM image of a small patch antenna and $\mathbf{b}$ the corresponding height profiles. c Electron-multiplying charge-coupled device (EMCCD) camera image of the radiation pattern of the same antenna measured in the far field by imaging the Fourier plane of the QD emission and $\mathbf{d}$ the associated polar plot. $\mathbf{e}(\theta, \varphi)$ polar plot of the numerically simulated emission pattern of a similar elliptical patch antenna and $\mathbf{f}$ the polar plot along the maximum intensity axis

atomic force microscopy (AFM) image above Fig. 2e shows an antenna patch after the final lift-off step.

This laser etching technique has several advantages over previous methods. Standard optical in situ lithography would require intense UV laser light for the exposure, which would degrade the QDs due to excessive light absorption. Here, by using a selected wavelength of 550-605 nm for the laser etching, we avoid such a detrimental effect. Moreover, this technique allows one to scan and locate the emitter without exposing the photoresist. Finally, as the etching process is highly nonlinear with respect to the excitation power, subwavelength structures can be obtained. The AFM image and height profile of the antenna patch in Fig. 3a, b, respectively, show a nanoantenna with dimensions as small as $200 \times 450 \mathrm{~nm}^{2}$, while the mean wavelength of the etching laser is $580 \mathrm{~nm}$. As the technique is deterministic, it is possible to characterize the emitter (in terms of the emission decay, polarization response, photon correlation, and spectral response) before it is embedded in the antenna. By noting antibunching in the QD emission before the in situ lithography, it is assured that the subsequent antenna will contain only one QD. The power, wavelength and exposure time of the laser can be optimized to prevent the degradation of the QDs after a successful lithography process. For the QD batch discussed in the paper, we noted that more than $80 \%$ of the QDs survived the laser etching process, from which all of the QDs survived the patch deposition process. This protocol ensures that all the fabricated antennas are single-emitter antennas.

\section{Emission from a subwavelength antenna}

We study the emission pattern of the very small antenna (Fig. 3a, b) coupled to a single QD. Such subwavelength plasmonic patch antennas have been predicted to show a very strong Purcell effect and directive emission ${ }^{7,26}$. Simulation studies have shown that these antennas exhibit a higher $F_{\mathrm{P}}$ if the emitter dipole is perpendicular rather than parallel to the patch. Moreover, for a perpendicular dipole, by reducing the patch diameter and employing specific patch sizes, $F_{\mathrm{P}}$ can increase from 70 (patch diameter of $\sim 1.6 \mu \mathrm{m}$ ) to more than 160 (patch diameter of $\sim 0.15 \mu \mathrm{m})$. Therefore, the ability to reduce the antenna patch to a subwavelength size is a tool that can be used to control the emission acceleration.

Figure 3c, $d$ shows the radiation pattern of the antenna measured in the far field by Fourier plane imaging. The antenna radiates through a cone in the far field, centred around $45^{\circ}$. This measurement is in good agreement with the numerical simulation of an elliptical patch antenna using the Fourier modal method ${ }^{33-36}$ as depicted in Fig. $3 \mathrm{e}, \mathrm{f}$ for the resonance wavelength at $604 \mathrm{~nm}$.

A striking feature of the measured radiative pattern is its high symmetry in the far field. Indeed, if the emitter is slightly off-centred with respect to the antenna patch, the radiation pattern rapidly exhibits angular asymmetry ${ }^{7}$. 

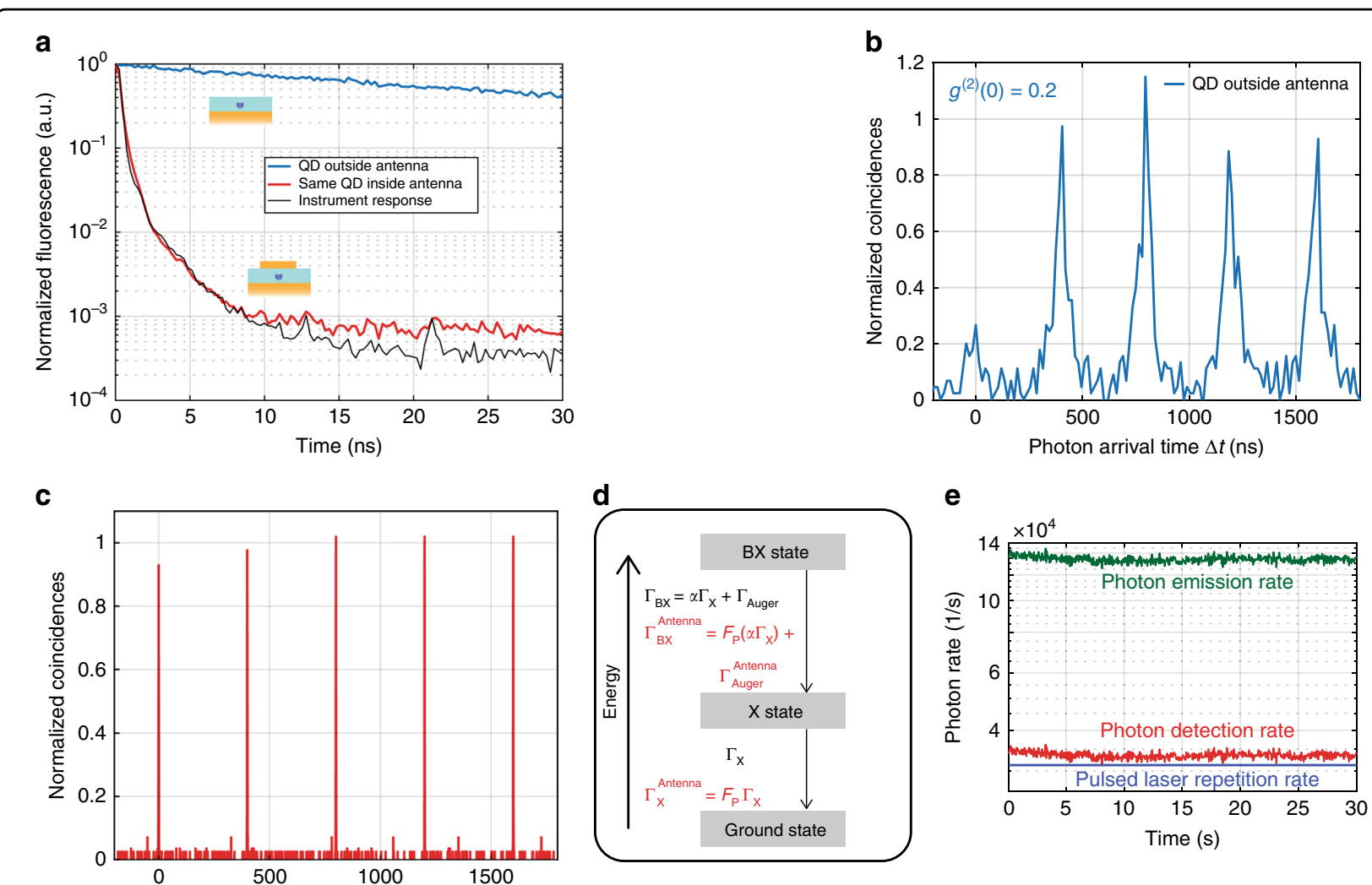

Fig. 4 Emission characteristics of a highly accelerated antenna. a Fluorescence decay of the QD before (blue line) and after (red line) it is placed inside the antenna. The instrument response function is shown in black. $\mathbf{b}$ Normalized photon coincidences measured for the QD before insertion in the antenna and $\mathbf{c}$ inside the antenna under a pulsed excitation of $0.03 \mathrm{~W} / \mathrm{cm}^{2}$ at $405 \mathrm{~nm}$. $\mathbf{d}$ QD energy-level diagram showing the biexciton (BX), exciton $(X)$, and ground states. e Detected photon rate (red) and the corresponding photon emission rate after the detection efficiency correction (green). The blue line denotes the pulsed laser repetition rate

The lobes of Fig. 3d, f demonstrate that the emitter is centred with respect to the antenna within $5 \mathrm{~nm}$, thus exhibiting the precision of the fabrication method used to position the QD laterally at the centre of the patch.

\section{Acceleration of spontaneous emission}

Figure 4a displays the fluorescence decay of a given QD before and after it is placed inside the patch antenna of Fig. 3. Before the deposition of the upper gold patch, when the QD is embedded in the PMMA layer and is $10 \mathrm{~nm}$ above the gold surface, its emission decay is monoexponential in character, which is indicative of singleexciton recombination with a decay of $\tau_{\mathrm{X}}^{\text {ref }}=36 \mathrm{~ns}$. After the deposition of a gold patch centred on this single QD, the emission lifetime is considerably reduced from $\tau_{\mathrm{X}}^{\text {ref }}$ to $\tau_{\mathrm{X}}^{\text {Antenna }}$, and the acceleration of the spontaneous emission $F_{\mathrm{P}}^{\text {Antenna }}=\frac{\tau_{\mathrm{X}}^{\text {Antenna }}}{\tau_{\mathrm{X}}^{\text {ref }}}$ of a given single QD can be measured. As the proximity to gold shortens the emitter lifetime ${ }^{37-40}$, we estimate that, on average, the lifetime $\tau_{\mathrm{X}}^{\text {ref }}$ of a single QD just above the gold (and not inside the antenna) is three times shorter than the decay time of the same colloidal QDs in a homogeneous PMMA layer, i.e., $\frac{\tau_{\mathrm{X}}^{\text {homogeneous }}}{\tau_{\mathrm{X}}^{\text {ref }}}=3$ (see Supplementary Information), where $\tau_{\mathrm{X}}^{\text {homogeneous }}$ is the exciton lifetime of the emitter in a homogeneous medium (infinite dielectric medium with a refractive index of 1.5, such as PMMA). The Purcell factor is calculated using the exciton decay rate and is given as $F_{\mathrm{P}}=\frac{\tau_{\mathrm{X}}^{\text {homogeneous }}}{\tau_{\mathrm{X}}^{\text {ref }}} \times \frac{\tau_{\mathrm{X}}^{\text {ref }}}{\tau_{\mathrm{X}}^{\text {Antenna }}}$.

Note that the acceleration of the spontaneous emission observed in Fig. 4a is so strong that we are limited by the response time of the measurement instrument to precisely quantify $F_{\mathrm{P}}$. By analysing the instrument response function (see Supplementary Information), we find that the emission acceleration is $>24$ with respect to the lifetime of the same QD before the gold patch deposition, which results in $F_{\mathrm{P}}>72$ (detailed in Supplementary Information), when compared with the case of a QD embedded in a homogeneous dielectric medium. We remark that the stated value of $F_{\mathrm{P}}$ was found by comparing the exciton 
lifetime of the QD with the slowest component of the instrument response function and not the faster component, resulting in a lower but more accurate value of $F_{\mathrm{P}}$. The method is detailed and justified in the Supplementary Information. In comparison to previous studies ${ }^{7,12,41}$, this extreme Purcell effect for a single emitter constitutes a high value that is obtained by the controlled and deterministic positioning of the emitter at the nanoscale in a highly confining subwavelength antenna structure.

\section{Quenching of the Auger processes and multiexciton emission}

As a consequence of such a significant Purcell effect, the electromagnetic decay channels are accelerated, making the radiative multiexciton recombination more efficient than the Auger non-radiative channels. The photon-correlation curve of Fig. 4b shows the measured second-order intensity correlation of the QD emission before it is placed in the antenna, exhibiting single-photon emission with $g^{(2)}(0)=$ 0.2 (calculated using a $15 \mathrm{~ns}$ binning interval). When inserted in the antenna, this value increases to $g^{(2)}(0)=1$ (Fig. 4c, calculated using a $0.5 \mathrm{~ns}$ binning interval), confirming the radiative multiexciton recombination.

Inside the plasmonic antenna, the radiative decay rates are accelerated by the Purcell factor $F_{\mathrm{P}}$ not only at the exciton level but also at the multiexciton level. As a result, the Auger processes that are very efficient at the multiexciton level become relatively inefficient in the highPurcell-factor regime, which facilitates the radiative recombination of the multiexcitons ${ }^{42-44}$. This is substantiated by the loss of single-photon emission in Fig. 4c. The three-level system in Fig. $4 \mathrm{~d}$ shows the different decay channels for a QD in a homogeneous medium (black colour) and the QD in an environment with a Purcell effect (red colour), such as a plasmonic antenna. In the homogeneous medium, the QD decays in a cascade from the biexciton $(\mathrm{BX})$ to the exciton $(\mathrm{X})$ state with a rate of $\Gamma_{\mathrm{BX}}=\alpha \Gamma_{\mathrm{X}}+\Gamma_{\text {Auger }}$ and from the exciton to the ground state with a rate of $\Gamma_{\mathrm{X}}$. When the same QD is placed in an environment such as a plasmonic antenna, due to the Purcell effect, the QD decays in a cascade from the biexciton to the exciton state with a rate of $\Gamma_{\mathrm{BX}}^{\text {Antenna }}=$ $F_{\mathrm{P}}\left(\alpha \Gamma_{\mathrm{X}}\right)+\Gamma_{\text {Auger }}^{\text {Antenna }}$ and from the exciton to the ground state with a rate of $F_{\mathrm{P}} \Gamma_{\mathrm{X}}$. For the homogeneous and antenna media, $\Gamma_{\mathrm{BX}}$ and $\Gamma_{\mathrm{BX}}^{\text {Antenna }}$ are the biexciton decay rates, $\Gamma_{X}$ and $\Gamma_{X}^{\text {Antenna }}$ are the exciton decay rates, and $\Gamma_{\text {Auger }}$ and $\Gamma_{\text {Auger }}^{\text {Antenna }}$ are the Auger decay rates, respectively. The Purcell factor $F_{\mathrm{P}}$ accelerates all channels except the Auger channels. The factor $\alpha \geq 2$ relates the biexciton and exciton decay rates ${ }^{45,46}$. To calculate $F_{\mathrm{P}}$, we use the experimentally measured $\Gamma_{\mathrm{X}}$ and $\Gamma_{\mathrm{X}}^{\text {Antenna }}$ and avoid comparing $\Gamma_{\mathrm{BX}}$ and $\Gamma_{\mathrm{BX}}^{\mathrm{Antenna}}$ to find $F_{\mathrm{P}}$ as this requires a separation of the Auger decay rates, which are not measured distinctively.

The plot of Fig. 4e illustrates very strong radiative multiexciton emission from a single-emitter plasmonic optical antenna. Under $405 \mathrm{~nm}$ pulsed excitation at a rate of $31.25 \mathrm{kHz}$ with a pulse width of $\sim 100 \mathrm{ps}$, the photon rate detected by the photodiodes is $\sim 34 \mathrm{kHz}$. Considering the $25 \%$ detection efficiency of the experimental setup, the photon emission rate from the QD is estimated to be $\sim 136 \mathrm{kHz}$. Therefore, for each laser pulse, the single emitter in the antenna emits $\sim 4-5$ photons. This demonstrates extremely efficient quenching of the Auger processes for at least 5 levels of radiative multiexciton recombination. Most likely, the relaxation of the QD inside the antenna includes many more higher multiexciton levels for which the non-radiative Auger recombination is not completely quenched, which therefore do not contribute to the radiation.

While a high Purcell effect in plasmonic antennas has often been associated with low brightness due to the predominance of non-radiative channels ${ }^{47-50}$, the structures developed here exhibit a very high fluorescence enhancement.
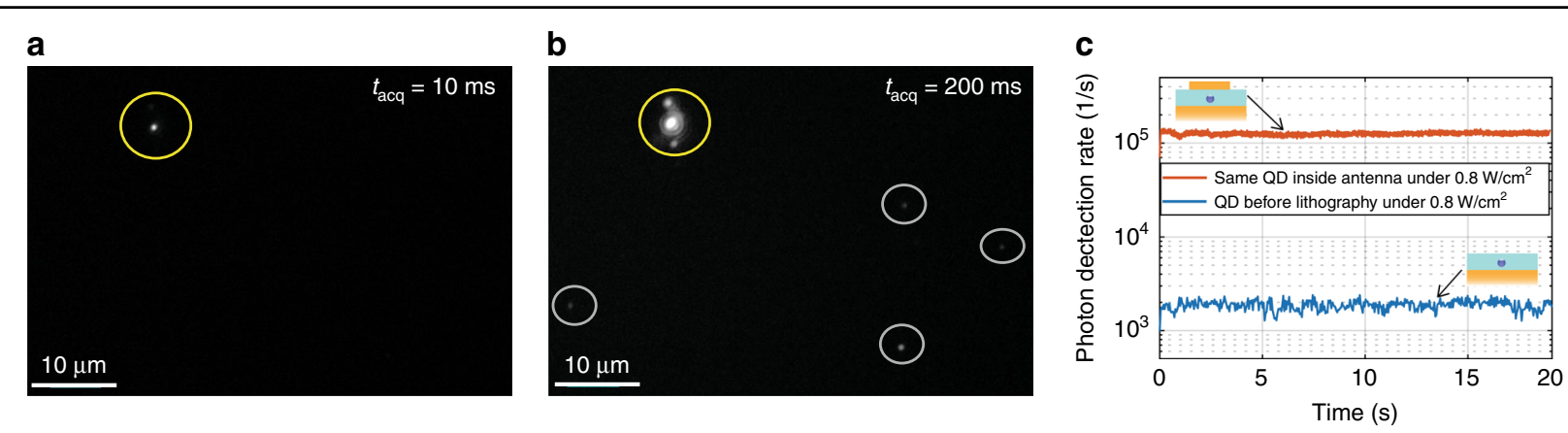

Fig. 5 Fluorescence enhancement by a plasmonic antenna. $\mathbf{a}, \mathbf{b}$ Fluorescence microscopy image of the antenna excited by a mercury lamp at $438 \pm 12 \mathrm{~nm}$ and captured by a CCD camera with an acquisition time of $10 \mathrm{~ms}$ (a) or $200 \mathrm{~ms}$ (b). The fluorescing antenna is encircled in yellow. Note that the two lighter spots above and below the saturated antenna spot in (b) are reflections on the protecting glass before the CCD camera sensor. c Photon detection rate from the QD before (blue) and after (red) it is placed inside the antenna. The antenna is excited at $405 \mathrm{~nm}$ with a pulsed laser at $2.5 \mathrm{MHz}$ 
Figure 5a, b are charge-coupled device (CCD) camera fluorescence images of the same area, including the small antenna of Fig. 3 and some QDs scattered outside the antenna. Under excitation with a lamp $(438 \pm 12 \mathrm{~nm})$, with an acquisition time of $10 \mathrm{~ms}$, only the antenna emission (indicated by the yellow circle in Fig. 5a) can be detected. An acquisition time of $200 \mathrm{~ms}$ (Fig. $5 \mathrm{~b}$ ) is required to detect the emission from the QDs outside antennas. To quantify the brightness of the antenna, we use a pulsed laser at $405 \mathrm{~nm}$ to excite the QD before and after insertion in the antenna. For the same excitation power, the antenna signal is 72 times more intense (Fig. 5c).

We also note the high optical quality and stability of the fabricated antenna. The antenna can sustain laser pulses with an instantaneous power of $120 \mathrm{~mW}$ for more than $10 \mathrm{~min}$ before it is photobleached-evidencing the quality of the QDs under study and their preservation during the laser etching protocol reported here. We finally note that the onset of multiexciton emission depends strongly on the antenna size and the Purcell effect due to the antenna. In general, the appearance of multiexciton emission depends on the relative weight of the radiative decay of the multiexciton states and the Auger rate. When increasing the number of excitons, the corresponding multiexciton emission rate increases. In the presence of efficient Auger processes, it is thus possible to observe multiexciton emission when increasing the excitation power to the extent that the multiexciton radiative decay rate exceeds the Auger rate. We have observed this effect studying several antennas of different Purcell factors. For larger (hence slower) antennas, the onset of multiexciton emission is typically observed at a larger excitation power than for smaller (hence faster) antennas. This effect can be used in applications that demand switching from single-photon emission to multiphoton emission ${ }^{51}$. Controlled roomtemperature multiexciton radiation can benefit photonic technologies $^{52}$ and improve the efficiency of lightemitting devices ${ }^{53,54}$.

\section{Discussion}

We have developed a novel deterministic laser etching protocol that enables the fabrication of subwavelength plasmonic antennas centred with a $50 \mathrm{~nm}$ lateral accuracy on a single colloidal QD. Due to the low mode volume and high field confinement, such an antenna exhibits a remarkable Purcell effect, directive emission and high brightness.

Further developments of the protocol include the selection of well-oriented emitters by polarization analysis ${ }^{49-51}$ for fully deterministic matching of the spectrum, location and orientation between the emitter and the antenna modes for optimized light-matter interactions. To reduce the losses in such structures, where more than $90 \%$ of the plasmonic energy can be lost as heat ${ }^{48}$, the fabrication capabilities of the protocol can be extended to produce hybrid structures such as metallo-dielectric antennas.

The same protocol can be used to synthesize other nanophotonic devices, plasmonic waveguides $^{55}$ and nanolasers ${ }^{56,57}$, which require deterministic and controlled positioning of a variety of sensitive emitters operating at room temperature, such as small colloidal QDs (e.g., CdSe/CdS and $\mathrm{PbSe} / \mathrm{PbS}$ ), nanodiamonds with vacancy centres such as nitrogen vacancies and silicon vacancies, and fluorescent molecules. Using this technique, while controlling the antenna shape and size and the dipolar orientation of the emitter inside the antenna and by using emitters with low multiexciton emission, very bright and directive single-photon sources based on such antennas can be obtained.

\section{Materials and methods \\ Sample preparation}

The CdSe cores of the colloidal CdSe/CdS core/shell QDs were synthesized using a protocol based on the work of Peng et $\mathrm{al}^{28}$, and the CdS shells were grown using the continuous slow injection method. On a $0.28-\mathrm{mm}$ thick polycrystalline Si substrate, a $200 \mathrm{~nm}$ layer of $\mathrm{Au}$ (refractive index of $n=0.0534+i 3.8249$ at $630 \mathrm{~nm}$ after deposition measured by ellipsometry) was grown by thermal evaporation using an adhesion layer of $\mathrm{Cr}(10 \mathrm{~nm})$. A $10-\mathrm{nm}$ thick film of polymethyl methacrylate (PMMA) was deposited on the Au substrate by spin-coating a solution of $0.5 \%[\mathrm{~m} / \mathrm{m}]$ PMMA (average molar mass of 101,000) in toluene at $4000 \mathrm{rpm}$ for $40 \mathrm{~s}$. The sample was baked at $150^{\circ} \mathrm{C}$ for $2 \mathrm{~min}$. Then, a dispersion of $\mathrm{CdSe} / \mathrm{CdS}$ QDs in hexane was spin-coated at $4000 \mathrm{rpm}$ for $40 \mathrm{~s}$ to obtain welldistributed individual QDs. Then, by spin-coating 1.5\% $[\mathrm{m} / \mathrm{m}]$ of PMMA solution in toluene at $4000 \mathrm{rpm}$ for $40 \mathrm{~s}$ on the QDs and baking the substrate at $150^{\circ} \mathrm{C}$ for $2 \mathrm{~min}$, individual QDs were embedded inside a dielectric layer of PMMA with $n=1.50$ at $630 \mathrm{~nm}$. To perform optical lithography on the sample, a lift-off resist (LOR $\left.{ }^{\circledR} 3 \mathrm{~A}\right)$ was spin-coated at $7000 \mathrm{rpm}$ for $40 \mathrm{~s}$, and the sample was baked at $150{ }^{\circ} \mathrm{C}$ for $2 \mathrm{~min}$, which produced a 300-nm thick layer. Then a 10-nm thick PMMA layer was deposited by spincoating a solution of $0.5 \%[\mathrm{~m} / \mathrm{m}]$ PMMA in toluene, and the sample was baked at $150^{\circ} \mathrm{C}$ for $2 \mathrm{~min}$.

\section{Lithography protocol}

The protocol has been described in the paper.

\section{Optical characterization and microscopy}

The sample was mounted on a PI P-713 XY Piezo Scanner nanopositioning stage and viewed with an inverted Olympus IX71 microscope using a 0.8NA 100× microscope air objective (Olympus LMPlanFL-100×). Light from a Hg lamp (Olympus USH-1030L) was filtered 
at $438 \pm 12 \mathrm{~nm}$ for widefield fluorescence imaging, and images were recorded by a CCD camera (Photonic Science CMOS). In a confocal setup, lifetime and photoncorrelation histograms were obtained using a $405 \mathrm{~nm}$ pulsed laser (pulse width of $100 \mathrm{ps)} \mathrm{at} 2.5 \mathrm{MHz}$ (PicoQuant LDH series), and optical lithography was performed by a supercontinuum laser (NKT Photonics SuperK Extreme with a SuperK Varia filter) as described previously. The emitted light was spectrally filtered by a $630 \pm 46 \mathrm{~nm}$ bandpass filter (Semrock 630/92 nm BrightLine $^{\circledR}$ ) and spatially filtered by a $150-\mu \mathrm{m}$ pinhole. Timeresolved photoluminescence and time-correlated singlephoton counting were performed using PicoHarp300 and two MPD PDM series single-photon avalanche diodes in a Hanbury-Brown and Twiss arrangement. The antenna emission pattern was recorded using a $0.95 \mathrm{NA} 100 \times$ microscope air objective (Olympus MPLAPON 100x) by Fourier plane imaging in the far field with an electronmultiplied CCD (EMCCD) camera (Andor iXon Ultra 897). The AFM images were recorded using a Veeco Dimension 3100 microscope with a Bruker RTESPA (Model: MPP-11120-10) tip in tapping mode. The transmission electron microscopy images were recorded with a Joel F2010 microscope.

\section{Acknowledgements}

The authors would like to thank Willy Daney de Marcillac for the spectrometric measurements, Loic Becerra, Bruno Gallas, Stéphane Chenot, and Stéphan Suffit for precious advice and help with the vapour deposition and ellipsometry, and Jean-Jacques Greffet and François Marquier for fruitful discussions. This work was supported by the regional funding DIM NanoK through the project PATCH and by the ANR DELIGHT.

\section{Author details \\ ${ }^{1}$ Institute of Fundamental and Frontier Sciences, University of Electronic Science and Technology of China, Chengdu 610054, People's Republic of China. ${ }^{2}$ Sorbonne Université, CNRS, Institut des Nanosciences de Paris, UMR 7588,75005 Paris, France. ${ }^{3}$ Centre de Nanosciences et de Nanotechnologies et de Nanostructures, CNRS UMR9001, Université Paris-Saclay, 10 boulevard Thomas Gobert, 91120 Marcoussis, France. ${ }^{4}$ Laboratoire de Physique et d'Etude des Matériaux, ESPCI-ParisTech, PSL Research University, Sorbonne Université, CNRS UMR 8213, 10 rue Vauquelin, Paris 75005, France. 'Laboratoire Charles Fabry, Institut d'Optique Graduate School, CNRS UMR 8501, Université Paris Saclay, 2 avenue Augustin Fresnel, 91127 Palaiseau Cedex, France}

\section{Author contributions}

A.R.D., C.B., P.S. and A.M. conceived and developed the fabrication protocol, A.R.D. and J.U.E. performed the optical characterization, M.N. and B.D. synthesized the QDs, J.P.H. performed the simulations, A.R.D., A.M., C.S., L.C. and Z.W. investigated the optical characterization data, and A.R.D., A.M. and P.S. wrote the paper.

\section{Conflict of interest}

The authors declare that they have no conflict of interest.

Supplementary information is available for this paper at https://doi.org/ 10.1038/s41377-020-0269-0.

Received: 5 November 2019 Revised: 28 January 2020 Accepted: 19 February 2020

Published online: 04 March 2020

\section{References}

1. Tame, M. S. et al. Quantum plasmonics. Nat. Phys. 9, 329-340 (2013).

2. Aharonovich, I., Englund, D. \& Toth, M. Solid-state single-photon emitters. Nat. Photonics 10, 631-641 (2016).

3. Gérard, J. M. et al. Enhanced spontaneous emission by quantum boxes in a monolithic optical microcavity. Phys. Rev. Lett. 81, 1110-1113 (1998).

4. Solomon, G. S., Pelton, M. \& Yamamoto, Y. Modification of spontaneous emission of a single quantum dot. Phys. Status Solidi (A) 178, 341-344 (2000).

5. Lodahl, P. et al. Controlling the dynamics of spontaneous emission from quantum dots by photonic crystals. Nature 430, 654-657 (2004).

6. Kinkhabwala, A. et al. Large single-molecule fluorescence enhancements produced by a bowtie nanoantenna. Nat. Photonics 3, 654-657 (2009).

7. Belacel, C. et al. Controlling spontaneous emission with plasmonic optical patch antennas. Nano Lett. 13, 1516-1521 (2013).

8. Bernal Arango, F., Kwadrin, A. \& Koenderink, A. F. Plasmonic antennas hybridized with dielectric waveguides. ACS Nano 6, 10156-10167 (2012).

9. Patel, S. K. \& Argyropoulos, C. Plasmonic nanoantennas: enhancing lightmatter interactions at the nanoscale. EPJ Appl. Metamaterials 2, 4 (2015).

10. Giannini, V. et al. Plasmonic nanoantennas: fundamentals and their use in controlling the radiative properties of nanoemitters. Chem. Rev. 111, 3888-3912 (2011).

11. Senellart, P., Solomon, G. \& White, A. High-performance semiconductor quantum-dot single-photon sources. Nat. Nanotechnol. 12, 1026-1039 (2017).

12. Ji, B. T. et al. Non-blinking quantum dot with a plasmonic nanoshell resonator. Nat. Nanotechnol. 10, 170-175 (2015).

13. Chikkaraddy, R. et al. Single-molecule strong coupling at room temperature in plasmonic nanocavities. Nature 535, 127-130 (2016).

14. Aharonovich, I., Greentree, A. D. \& Prawer, S. Diamond photonics. Nat. Photonics 5, 397-405 (2011).

15. Ureña, E. B. et al. Excitation enhancement of a quantum dot coupled to a plasmonic antenna. Adv. Mater. 24, OP314-OP320 (2012).

16. Hoang, T. B., Akselrod, G. M. \& Mikkelsen, M. H. Ultrafast room-temperature single photon emission from quantum dots coupled to plasmonic nanocavities. Nano Lett. 16, 270-275 (2016).

17. Le-Van, Q. et al. Temperature dependence of quantum dot fluorescence assisted by plasmonic nanoantennas. Phys. Rev. B 91, 085412 (2015).

18. Hoang, T. B. et al. Ultrafast spontaneous emission source using plasmonic nanoantennas. Nat. Commun. 6, 7788 (2015).

19. Vahala, K. J. Optical microcavities. Nature 424, 839-846 (2003).

20. Dousse, A. et al. Controlled light-matter coupling for a single quantum dot embedded in a pillar microcavity using far-field optical lithography. Phys. Rev. Lett. 101, 267404 (2008).

21. Somaschi, N. et al. Near-optimal single-photon sources in the solid state. Nat. Photonics 10, 340-345 (2016).

22. Gazzano, O. et al. Evidence for confined Tamm plasmon modes under metallic microdisks and application to the control of spontaneous optical emission. Phys. Rev. Lett. 107, 247402 (2011).

23. Gschrey, M. et al. Highly indistinguishable photons from deterministic quantum-dot microlenses utilizing three-dimensional in situ electron-beam lithography. Nat. Commun. 6, 7662 (2015).

24. Purcell, E. M. in Confined Electrons and Photons (eds Burstein, E. \& Weisbuch, C.) 839 (Springer, Boston, MA, 1995).

25. Grynberg, G., Aspect, A. \& Fabre, C. Introduction to Quantum Optics: From the Semi-classical Approach to Quantized Light. (Cambridge University Press, Cambridge, 2010).

26. Esteban, R., Teperik, T. V. \& Greffet, J. J. Optical patch antennas for single photon emission using surface plasmon resonances. Phys. Rev. Lett. 104 026802 (2010).

27. Novotny, L. \& Van Hulst, N. Antennas for light. Nat. Photonics 5, 83-90 (2011).

28. Peng, X. G., Wickham, J. \& Alivisatos, A. P. Kinetics of II-VI and III-V colloidal semiconductor nanocrystal growth: "Focusing" of size distributions. J. Am. Chem. Soc. 120, 5343-5344 (1998).

29. Reiss, P., Protière, M. \& Li, L. Core/shell semiconductor nanocrystals. Small 5, 154-168 (2009).

30. Klimov, V. I. Nanocrystal Quantum Dots. 2nd edn (CRC Press, Boca Raton, 2010).

31. Mahler, B. et al. Towards non-blinking colloidal quantum dots. Nat. Mater. 7, 659-664 (2008).

32. Forsén, E. et al. Laser lithography on resist bi-layer for nanoelectromechanical systems prototyping. Microelectron. Eng. 73-74, 491-495 (2004). 
33. Yang, J. J., Hugonin, J. P. \& Lalanne, P. Near-to-far field transformations for radiative and guided waves. ACS Photonics 3, 395-402 (2016).

34. Hugonin, J. \& Lalanne, P. Perfectly matched layers as nonlinear coordinate transforms: a generalized formalization. J. Optical Soc. Am. A 22, 1844-1849 (2005).

35. Bai, Q. et al. Efficient and intuitive method for the analysis of light scattering by a resonant nanostructure. Opt. Express 21, 27371-27382 (2013).

36. David, A., Benisty, H. \& Weisbuch, C. Fast factorization rule and plane-wave expansion method for two-dimensional photonic crystals with arbitrary holeshape. Phys. Rev. B 73, 075107 (2006).

37. Vion, C. et al. Controlled modification of single colloidal CdSe/ZnS nanocrystal fluorescence through interactions with a gold surface. Opt. Express 18, 7440-7455 (2010)

38. Chance, R. R., Prock, A. \& Silbey, R. Comments on the classical theory of energy transfer. J. Chem. Phys. 62, 2245-2253 (1975).

39. Novotny, L. \& Hecht, B. Principles of Nano-Optics. 2nd edn (Cambridge University Press, Cambridge, 2012)

40. Lukosz, W. Theory of optical-environment-dependent spontaneous-emission rates for emitters in thin layers. Phys. Rev. B 22, 3030-3038 (1980).

41. Canneson, D. et al. Enhancing the fluorescence of individual thick shell CdSe/CdS nanocrystals by coupling to gold structures. N. J. Phys. 14, 063035 (2012)

42. Naiki, H. et al. Single-photon emission behavior of isolated cdse/zns quantum dots interacting with the localized surface plasmon resonance of silver nanoparticles. J. Phys. Chem. C. 115, 23299-23304 (2011).

43. LeBlanc, S. J. et al. Enhancement of multiphoton emission from single CdSe quantum dots coupled to gold films. Nano Lett. 13, 1662-1669 (2013).

44. Park, Y. S. et al. Super-poissonian statistics of photon emission from single CdSe-CdS core-shell nanocrystals coupled to metal nanostructures. Phys. Rev. Lett. 110, 117401 (2013).
45. Nair, G. \& Bawendi, M. G. Carrier multiplication yields of CdSe and CdTe nanocrystals by transient photoluminescence spectroscopy. Phys. Rev. B 76 081304 (2007).

46. Nair, G., Zhao, J. \& Bawendi, M. G. Biexciton quantum yield of single semiconductor nanocrystals from photon statistics. Nano Lett. 11, 1136-1140 (2011).

47. Ozbay, E. Plasmonics: merging photonics and electronics at nanoscale dimensions. Science 311, 189-193 (2006).

48. Bigourdan, F. et al. Design of highly efficient metallo-dielectric patch antennas for single-photon emission. Opt. Express 22, 2337-2347 (2014).

49. Markel, V. A. \& Sarychev, A. K. Propagation of surface plasmons in ordered and disordered chains of metal nanospheres. Phys. Rev. B 75, 085426 (2007).

50. Kuttge, M. et al. Loss mechanisms of surface plasmon polaritons on gold probed by cathodoluminescence imaging spectroscopy. Appl. Phys. Lett. 93 113110 (2008).

51. Kambhampati, P. Multiexcitons in semiconductor nanocrystals: a platform for optoelectronics at high carrier concentration. J. Phys. Chem. Lett. 3, 1182-1190 (2012).

52. Fisher, B. et al. Multiexciton fluorescence from semiconductor nanocrystals. Chem. Phys. 318, 71-81 (2005).

53. Dang, C. et al. Red, green and blue lasing enabled by single-exciton gain in colloidal quantum dot films. Nat. Nanotechnol. 7, 335-339 (2012).

54. Malko, A. V. et al. From amplified spontaneous emission to microring lasing using nanocrystal quantum dot solids. Appl. Phys. Lett. 81, 1303-1305 (2002).

55. Kumar, S. \& Bozhevolnyi, S. I. Excitation of hybrid plasmonic waveguide modes by colloidal quantum dots. ACS Photonics 6, 1587-1593 (2019).

56. Oulton, R. F. et al. Plasmon lasers at deep subwavelength scale. Nature 461, 629-632 (2009).

57. Sidiropoulos, T. P. H. et al. Ultrafast plasmonic nanowire lasers near the surface plasmon frequency. Nat. Phys. 10, 870-876 (2014). 